\title{
Two black hole initial data
}

\author{
Szymon Łęski \\ Centrum Fizyki Teoretycznej \\ Polska Akademia Nauk \\ Al. Lotników 32/46, Warsaw, Poland
}

\begin{abstract}
The Misner initial data are a standard example of time-symmetric initial data with two apparent horizons. Compact formulae describing such data are presented in the cases of equal or nonequal masses (i.e. isometric or non-isometric horizons). The interaction energy in the "Schwarzschild + test particle" limit of the Misner data is analyzed.
\end{abstract}

PACS numbers: 04.20.Ex, 04.70.Bw

\section{INTRODUCTION}

In 1] Misner proposed two-body wormhole initial data and in 2] he used the method of images to describe the time-symmetric initial data for an arbitrary number $N$ of particles (see also [3] for a review of time-symmetric initial data). Such data may be viewed as a collection of Einstein-Rosen bridges connecting two isometric sheets. If we restrict ourselves to just one of them, we can view such initial data as a three-dimensional metric on a manifold with internal boundary. The boundary consists of $N$ minimal surfaces representing surfaces of the "black holes" corresponding to the particles. In this paper I am concerned with the $N=2$ case only. If the masses of black holes are equal (i.e. when the horizons are isometric) then there is a well-known formula for the three-metric:

$$
g=\Phi^{4}\left(\mathrm{~d} \mu^{2}+\mathrm{d} \eta^{2}+\sin ^{2} \eta \mathrm{d} \varphi^{2}\right),
$$

where

$$
\Phi=\sum_{n \in \mathbb{Z}} \frac{\sqrt{d}}{\sqrt{\cosh \left(\mu+2 n \mu_{0}\right)-\cos \eta}} .
$$

Here $(\mu, \eta, \varphi)$ are the bispherical coordinates defined by

$$
\begin{aligned}
& x=\cos \varphi \frac{\sin \eta}{\cosh \mu-\cos \eta}, \\
& y=\sin \varphi \frac{\sin \eta}{\cosh \mu-\cos \eta}, \\
& z=\frac{\sinh \mu}{\cosh \mu-\cos \eta} .
\end{aligned}
$$

There are two parameters in (2): $d$ and $\mu_{0}$. The $\mu$ coordinate ranges from $-\mu_{0}$ to $\mu_{0}$. The $\mu= \pm \mu_{0}$ surfaces are minimal, which translates to the Neumann boundary condition for $\Phi:\left.\frac{\partial}{\partial \mu} \Phi\right|_{\mu= \pm \mu_{0}}=0$. The conformal factor $\Phi$ satisfies an elliptic equation

$$
\sin \eta\left(\Delta-\frac{1}{4}\right) \Phi=-4 \pi \sqrt{d} \delta_{0},
$$

where $\Delta$ is the Beltrami-Laplace operator associated with the metric $\mathrm{d} \mu^{2}+\mathrm{d} \eta^{2}+\sin ^{2} \eta \mathrm{d} \varphi^{2}$ and $\sin \eta$ is the volume element.

It is obvious that the metric $g$ is conformally related to the Euclidean metric on $\mathbb{R}^{3}$ minus two balls which correspond to $|\mu|>\mu_{0}$. This transformation reads:

$$
g=\Psi^{4} d^{2}\left(\mathrm{~d} x^{2}+\mathrm{d} y^{2}+\mathrm{d} z^{2}\right)
$$

*Electronic address: szleski@cft.edu.pl 
where the conformal factor $\Psi$ is related to $\Phi$ by the formula $\Psi=d^{-1 / 2} \Phi \sqrt{\cosh \mu-\cos \eta}$. The $\sqrt{d}$ factor is extracted from $\Phi$ in order to normalize the value of $\Psi$ at infinity to 1 . Now the parameter $2 d$ has the interpretation of the Euclidean distance (with respect to the metric $d^{2}\left(\mathrm{~d} x^{2}+\mathrm{d} y^{2}+\mathrm{d} z^{2}\right)$ ) between the points corresponding to $\mu= \pm \infty$ $(z= \pm 1)$.

To generalize the metric $g$ to the case of nonequal masses one takes $\mu \in[-a, b]$ with $a, b>0$, imposes the Neumann boundary conditions and solves equation (4) for $\Phi$. Such metrics are well known and used to construct initial data. There are effective formulae which give the conformal factor, see for example [4]. However, it seems that no closedform formula has been published, for example the formulae from [2] include series of operators of inversion with respect to spheres in Euclidean space and the formulae in [4] are recursive. Below I present a compact form of the metric. First I derive a new version of formula (2) in the equal-mass case, see (7) and (8). Then I present the corresponding formulae (10) and (11) in the case of nonequal masses. I also use (11) to calculate the interaction energy in the test-body limit of the Misner initial data, that means when one mass is much smaller than the other.

\section{MAIN RESULT}

Let us begin with

$$
\frac{1}{\left|\vec{r}-\vec{r}^{\prime}\right|}=\sum_{l=0}^{\infty} \frac{r_{<}^{l}}{r_{>}^{l+1}} P_{l}(\cos \beta)
$$

where $r_{<}:=\min \left(|\vec{r}|,\left|\vec{r}^{\prime}\right|\right), r_{>}:=\max \left(|\vec{r}|,\left|\vec{r}^{\prime}\right|\right), \beta$ - the angle between $\vec{r}, \vec{r}^{\prime}$. Taking $\vec{r}^{\prime}=\vec{e}_{z}$ and $\left|\vec{r}^{\prime}\right|=\exp (\alpha)$, we get

$$
\left|\vec{e}_{z}-\vec{r}^{\prime}\right|=\sqrt{2} e^{\alpha / 2} \sqrt{\cosh \alpha-\cos \eta},
$$

where $\eta$ is a spherical angle of the vector $\vec{r}^{\prime}$. Hence we have the following formula for terms in (2):

$$
\frac{1}{\sqrt{\cosh \alpha-\cos \eta}}=\sqrt{2} \sum_{l=0}^{\infty} e^{-|\alpha|(l+1 / 2)} P_{l}(\cos \eta)
$$

where $\alpha \neq 0$. If we expand the summands in (2) using (6) and sum the $n$-indexed series then we arrive at the conformal factor (2) for the equal-mass case rewritten as:

$$
\Phi=\sqrt{2 d} \sum_{l=0}^{\infty} P_{l}(\cos \eta) \frac{\cosh \left[\left(\mu_{0}-|\mu|\right)(l+1 / 2)\right]}{\sinh \left[\mu_{0}(l+1 / 2)\right]}
$$

the formula being valid for $\mu \neq 0$. We can also extract the singular term $\frac{\sqrt{d}}{\sqrt{\cosh \mu-\cos \eta}}$ and get the following version of (71):

$$
\begin{aligned}
\Phi & =\frac{\sqrt{d}}{\sqrt{\cosh \mu-\cos \eta}} \\
& +\sqrt{2 d} \sum_{l=0}^{\infty} P_{l}(\cos \eta) e^{-\mu_{0}(l+1 / 2)} \frac{\cosh [\mu(l+1 / 2)]}{\sinh \left[\mu_{0}(l+1 / 2)\right]}
\end{aligned}
$$

with the series being uniformly convergent for $\mu \in\left[-\mu_{0}, \mu_{0}\right]$.

In the case of non-equal masses the conformal factor is given by the following formula:

$$
\Phi=\frac{\sqrt{d}}{\sqrt{\cosh \mu-\cos \eta}}+\sqrt{d} \sum_{n=1}^{\infty}\left(Q_{-2 n a-2(n-1) b}+Q_{-2 n a-2 n b}+Q_{2(n-1) a+2 n b}+Q_{2 n a+2 n b}\right)
$$

where

$$
Q_{\alpha}:=\frac{1}{\sqrt{\cosh \alpha-\cos \eta}} .
$$

Simple symmetry argument shows that such $\Phi$ indeed satisfies the Neumann boundary conditions at $\mu=-a, \mu=b$. As before we use (6) and perform summation over $n$ to get $\Phi$ in the following compact form:

$$
\Phi=\sqrt{2 d} \sum_{l=0}^{\infty} P_{l}(\cos \eta) \frac{\cosh [(a-b+\mu)(l+1 / 2)]+\cosh [(a+b-|\mu|)(l+1 / 2)]}{\sinh [(a+b)(l+1 / 2)]}
$$


or, if we extract the singular term,

$$
\begin{aligned}
\Phi & =\frac{\sqrt{d}}{\sqrt{\cosh \mu-\cos \eta}}+ \\
& \sqrt{2 d} \sum_{l=0}^{\infty} P_{l}(\cos \eta) \frac{e^{-\mu(l+1 / 2)}\left(e^{2 b(l+1 / 2)}+1\right)+e^{\mu(l+1 / 2)}\left(e^{2 a(l+1 / 2)}+1\right)}{e^{2(a+b)(l+1 / 2)}-1} .
\end{aligned}
$$

Again formula (10) is valid for $\mu \neq 0$ and the series in (11) converges uniformly for $\mu \in[-a, b]$. If we substitute $a=b=\mu_{0}$ then (10) and (11) reduce to (7) and (8), respectively.

The main result here are formulae (10) and (11). It is worth noting that the conformal factor is expanded in a series of orthogonal polynomials, which is a desirable feature for numerical treatment.

\section{SCHWARZSCHILD + TEST-BODY LIMIT}

Let us now analyze the "Schwarzschild + test body" limit of Misner initial data. First, we observe that if we pass to the limit $b \rightarrow \infty$ in (11) then we get Schwarzschild initial data in bispherical coordinates. The $\mu=-a$ sphere represents the minimal surface in Schwarzschild initial data and $\mu=\infty$ becomes a regular point. The mass of such data is

$$
m=\frac{2 d}{\sinh a} .
$$

Second, we treat $\varepsilon:=\exp (-b)$ as a small parameter, that means $\varepsilon \ll \exp (-a)$, and expand (11) in $\varepsilon$. We get the following formula for the conformal factor:

$$
\begin{aligned}
\Phi & =\frac{\sqrt{d}}{\sqrt{\cosh \mu-\cos \eta}} \\
& +\sqrt{2 d} \sum_{l=0}^{\infty} \frac{e^{-\mu(l+1 / 2)}}{e^{2 a(l+1 / 2)}} P_{l}(\cos \eta)+\left(1+e^{-a}\right)\left(e^{\mu / 2}+e^{-\mu / 2-a}\right) \varepsilon+O\left(\varepsilon^{2}\right)
\end{aligned}
$$

Let us denote by $m_{1}$ and $m_{2}$ the masses of the $\mu=-a$ and the $\mu=b$ surfaces respectively. By "mass of a surface" I mean here the square root of its area divided by $\sqrt{16 \pi}$. Using (13) we find the individual masses of the surfaces and the ADM mass $M$ of the whole system:

$$
\begin{gathered}
m_{1}=\frac{2 d}{\sinh a}+8 d\left(e^{-a}+e^{-2 a}\right) \varepsilon+O\left(\varepsilon^{2}\right) \\
m_{2}=4 d\left(1+e^{-a}\right)^{2} \varepsilon+O\left(\varepsilon^{2}\right) \\
M=m_{1}+m_{2}-8 d\left(e^{-a}+e^{-2 a}\right) \varepsilon+O\left(\varepsilon^{2}\right) .
\end{gathered}
$$

In the Newtonian gravity the interaction energy equals $\stackrel{\circ}{E}=-\frac{m_{1} m_{2}}{\text { distance }}$ and for the Misner data the interaction energy should converge to this value in the Newtonian limit, that means when the distance increases to infinity. We would like to write the interaction energy in the form $E=-\frac{m_{1} m_{2}}{\text { distance }} \gamma$, where $\gamma$ is a function of distance and converges to 1 as the distance goes to infinity. The two most obvious choices for the distance parameter are $D:=2 d$ and the length of the $\eta=\pi$ geodesic connecting two horizons which we denote by $L$. The distance $D$ is quite close to the distance studied by Brill and Lindquist [ $\underline{5}$, in the sense that it is the Euclidean distance between two points lying inside minimal surfaces. In this case we get the following formula for the interaction energy:

$$
M-m_{1}-m_{2}=-\frac{m_{1} m_{2}}{D}\left(1-\frac{\frac{m_{1}}{D}}{1+\sqrt{1+\left(\frac{m_{1}}{D}\right)^{2}}}\right)+O\left(\varepsilon^{2}\right) .
$$

Passing now to the second case, we can calculate the geodesic distance $L$ as the integral

$$
L=d \int_{z_{1}}^{1}(\Psi(x=0, y=0, z))^{2} \mathrm{~d} z+O(\varepsilon)
$$


where $z_{1}=-\frac{\sinh a}{\cosh a+1}$ is the $z$ coordinate (3) of the point $(\mu=-a, \eta=\pi)$. The result is

$$
L=2 d\left[\frac{1}{2}-\frac{z_{1}}{2}+\frac{m_{1}}{2 d} \log \frac{1-z_{0}}{z_{1}-z_{0}}+\frac{m_{1}^{2}}{8 d^{2}}\left(\frac{1}{z_{1}-z_{0}}-\frac{1}{1-z_{0}}\right)\right]+O(\varepsilon)
$$

with $z_{0}=-\frac{\cosh a}{\sinh a}$ being the $z$ coordinate of the center of the removed ball corresponding to the $\mu=-a$ minimal surface. As the result we get the interaction energy equal to

$$
M-m_{1}-m_{2}=-\frac{m_{1} m_{2}}{L} \gamma_{L}+O\left(\varepsilon^{2}\right) .
$$

The factor

$$
\gamma_{L}:=\frac{L}{2 d}\left(1-\frac{\frac{m_{1}}{2 d}}{1+\sqrt{1+\left(\frac{m_{1}}{2 d}\right)^{2}}}\right)
$$

can be expressed in zeroth order in $\varepsilon$ (using (14) ) as a rather complicated function of a single parameter $\frac{2 d}{m_{1}}$. In the $d \rightarrow \infty$ limit $(L \rightarrow \infty)$ it converges to 1 , giving the correct Newtonian limit of (18).

\section{ACKNOWLEDGMENTS}

This research was supported by the Polish Research Council grant KBN 2 P03B 07324 and by the Erwin Schrödinger Institute.

[1] C. W. Misner, Phys. Rev. 118, 1110 (1960).

[2] C. W. Misner, Ann. Phys. (NY) 24, 102 (1963).

[3] D. Giulini, in Black Holes: Theory and Observation (Springer Verlag, Berlin, 1998), URL gr-qc/9804055

[4] G. B. Cook, Phys. Rev. D 44, 2983 (1991).

[5] D. R. Brill and R. W. Lindquist, Phys. Rev. 131, 471 (1963). 